Ferrata Storti Foundation

\title{
Impact of induction regimen and allogeneic hematopoietic cell transplantation on outcome in younger adults with acute myeloid leukemia with a monosomal karyotype
}

Haematologica 2019

Volume 104(6):1168-1175

\section{Correspondence:}

FRÉDÉRIC BARON

f.baron@ulg.ac.be

Received: August 17, 2018.

Accepted: November 29, 2018.

Pre-published: December 6, 2018.

doi:10.3324/haematol.2018.204826

Check the online version for the most updated information on this article, online supplements, and information on authorship \& disclosures: www.haematologica.org/content/104/6/1168

\section{(C)2019 Ferrata Storti Foundation}

Material published in Haematologica is covered by copyright. All rights are reserved to the Ferrata Storti Foundation. Use of published material is allowed under the following terms and conditions:

https://creativecommons.org/licenses/by-nc/4.0/legalcode. Copies of published material are allowed for personal or internal use. Sharing published material for non-commercial purposes is subject to the following conditions: https://creativecommons.org/licenses/by-nc/4.0/legalcode, sect. 3. Reproducing and sharing published material for commercial purposes is not allowed without permission in writing from the publisher.

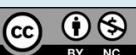

Frédéric Baron, ${ }^{1}$ Marian Stevens-Kroef, ${ }^{2}$ Michal Kicinski, ${ }^{3}$ Giovanna Meloni, ${ }^{4}$ Petra Muus, ${ }^{2,5}$ Jean-Pierre Marie,${ }^{6}$ Constantijn J.M. Halkes, ${ }^{7}$ Xavier Thomas, ${ }^{8}$ Radovan Vrhovac, ${ }^{9}$ Francesco Albano, ${ }^{10}$ François Lefrère Sr., ${ }^{11}$ Simona Sica, ${ }^{12}$ Marco Mancini, ${ }^{4}$ Adriano Venditti, ${ }^{13}$ Anne Hagemeijer, ${ }^{14}$ Joop H. Jansen, ${ }^{2}$ Sergio Amadori, ${ }^{13}$ Theo de Witte, ${ }^{2}$ Roelof Willemze ${ }^{7}$ and Stefan Suciu ${ }^{3}$

${ }^{1}$ Groupe Interdisciplinaire de Génoprotéomique Appliquée (GIGA), Laboratory of Hematology, University of Liege, Belgium; ${ }^{2}$ Radboud University Medical Center, Nijmegen, the Netherlands; ${ }^{3}$ EORTC Headquarters, Brussels, Belgium; ${ }^{4}$ Department of Hematology, Sapienza University, Rome, Italy; ${ }^{5}$ King's College Hospital, London, UK; ${ }^{6}$ Department of Hematology, Saint Antoine Hospital, Paris, France; ' 7 Leiden University Medical Center, the Netherlands; ${ }^{8} \mathrm{CHU}$ Lyon, France; ${ }^{9}$ University Hospital Center Zagreb, Croatia; ${ }^{10}$ University of Bari, Italy; ${ }^{11}$ Necker Hospital, Paris, France; ${ }^{12}$ Università Cattolica Sacro Cuore, Roma, Italy; ${ }^{13}$ University Tor Vergata, Roma, Italy and ${ }^{14}$ University of Leuven, Belgium

\section{ABSTRACT}

M onosomal karyotype confers a poor prognosis in patients with acute myeloid leukemia. Here, we determined the impact of the type of remission-induction chemotherapy and the impact of having a donor in younger acute myeloid leukemia patients with a monosomal karyotype included in two phase III trials. In the first trial patients were randomized to receive either daunorubicin, mitoxantrone, or idarubicin in addition to standard-dose cytarabine and etoposide for induction chemotherapy. In the second trial patients were randomized to standarddose cytarabine or high-dose cytarabine induction, both with daunorubicin and etoposide. In both trials, patients who achieved a complete remission with or without complete hematologic recovery underwent allogeneic hematopoietic stem cell transplantation if they had a donor; otherwise, they underwent autologous transplantation. In comparison to patients with intermediate-risk cytogenetics without a monosomal karyotype $(n=1,584)$ and with adverse cytogenetics without a monosomal karyotype $(n=218)$, patients with a monosomal karyotype $(n=188)$ were more likely not to achieve a complete remission with or without count recovery [odds ratio $=2.85,95 \%$ confidence interval $(95 \%, \mathrm{CI}): 2.10-3.88]$ and had shorter overall survival [hazard ratio, $(\mathrm{HR})=2.44,95 \% \mathrm{CI}$ : $2.08-2.88$ ]. There was no impact of the type of anthracycline or of the dose of cytarabine on outcomes in patients with a monosomal karyotype. Among monosomal karyotype patients who achieved a complete remission with or without count recovery, HLA-identical related donor availability was associated with longer survival from complete remission with or without count recovery ( $\mathrm{HR}=0.59$, 95\% CI: 0.37-0.95). ClinicalTrials.gov identifiers: AML-10: NCT00002549; AML-12: NCT00004128.

\section{Introduction}

The prognosis of young adult patients with intermediate/high-risk acute myelogenous leukemia (AML) remains unsatisfactory. With current remission induction chemotherapy, $15-40 \%$ of such patients fail to achieve a complete remission (CR), and only $30-50 \%$ of them remain alive for more than 5 years..$^{1-4}$ Approximately $55 \%$ of AML patients have at least one chromosomal abnormali- 
ty that can be detected by conventional cytogenetics. ${ }^{5}$ In younger AML patients, the karyotype of leukemic cells has remained one of the main prognostic factors. ${ }^{5,6}$ Specifically, patients can be classified into a favorable, intermediate or adverse group. 5,6 This classification not only provides information on prognosis, but also influences the choice of post-remission treatment., In 2008, Breems et al. identified a group of cytogenetic abnormalities, termed monosomal karyotype (MK), which was associated with a particularly poor prognosis. ${ }^{8} \mathrm{MK}$ is defined as the presence of two or more autosomal monosomies, or a single monosomy in the presence of structural abnormalities. .,9 $^{2}$

The aim of this study was to determine the impact of the type of remission-induction chemotherapy and allogeneic hematopoietic stem cell transplantation (HSCT) in younger AML patients with a MK, using the data from the European Organization for Research and Treatment on Cancer/Gruppo Italiano Malattie Ematologiche dell'Adulto (EORTC/GIMEMA) AML-10 and AML-12 phase III multicenter trials.

\section{Methods}

\section{Study design}

In the EORTC Leukemia Group/GIMEMA AML-10 trial, ${ }^{2}$ patients 15-60 years old were randomized to receive either daunorubicin $\left(50 \mathrm{mg} / \mathrm{m}^{2}\right)$, mitoxantrone $\left(12 \mathrm{mg} / \mathrm{m}^{2}\right)$, or idarubicin $\left(10 \mathrm{mg} / \mathrm{m}^{2}\right)$ on days 1,3 and 5 in addition to standard-dose cytarabine $\left(25 \mathrm{mg} / \mathrm{m}^{2}\right.$ bolus followed by $100 \mathrm{mg} / \mathrm{m}^{2}$ given as a continuous infusion daily for 10 days) and etoposide $\left(100 \mathrm{mg} / \mathrm{m}^{2}\right.$ on days 1-5) for induction chemotherapy.

In the EORTC Leukemia Group/GIMEMA AML-12 trial, ${ }^{1}$ patients 15-60 years old were randomized between standard-dose cytarabine induction: daunorubicin $\left(50 \mathrm{mg} / \mathrm{m}^{2}\right.$ per day on days 1 , 3 , and 5) plus etoposide (50 $\mathrm{mg} / \mathrm{m}^{2}$ per day on days $\left.1-5\right)$ plus 10 days of cytarabine $\left(100 \mathrm{mg} / \mathrm{m}^{2}\right.$ per day as a continuous intravenous infusion) and high-dose cytarabine induction: daunorubicin $\left(50 \mathrm{mg} / \mathrm{m}^{2}\right.$ on days 1,3 , and 5$)$ plus etoposide $\left(50 \mathrm{mg} / \mathrm{m}^{2}\right.$ per day on days $1-5)$ plus cytarabine $\left(3,000 \mathrm{mg} / \mathrm{m}^{2}\right.$ every $12 \mathrm{~h}$ as a $3 \mathrm{~h}$ intravenous infusion on days $1,3,5$, and 7 ).

In both trials, a second cycle of induction was administered to patients who achieved a partial response. Patients who achieved a CR or a CR with incomplete blood count recovery (CRi) after one or two courses of induction chemotherapy received consolidation chemotherapy with the same anthracycline as in the induction course plus intermediate-dose cytarabine $\left(500 \mathrm{mg} / \mathrm{m}^{2}\right.$ every $12 \mathrm{~h}$ as a $2 \mathrm{~h}$ intravenous infusion on days $1-6)$. Younger patients ( $<46$ years in AML-10 and $<50-60$ years in AML-12) were then scheduled to undergo allogeneic HSCT in first CR/CRi if they had an HLA-identical family donor (in both trials) or if they had an unrelated donor and needed two induction courses to achieve a CR/CRi or had chromosome abnormalities involving 3q, 5, 7, $11 \mathrm{q} 23, \mathrm{t}(6 ; 9), \mathrm{t}(9 ; 22)$ or complex abnormalities (in the AML-12 trial). Patients without a donor were scheduled to undergo autologous HSCT in first CR/CRi.

\section{Ethics approval and consent to participation}

This is a retrospective analysis limited to data from patients included in phase III multicenter prospective trials (either the EORTC/GIMEMA AML-10 or the EORTC/GIMEMA AML-12). Both prospective phase III trials were approved by the internal review boards of EORTC and GIMEMA and the ethical committee of each participating institution, and were conducted in accor- dance with the Declaration of Helsinki. All patients signed the respective informed consent form.

\section{Cytogenetic assessment}

Cytogenetic examinations were performed at diagnosis. Cytogenetic data were centrally reviewed. ${ }^{2}$ For the current analysis, cytogenetics were centrally re-reviewed, described according to International System for Cytogenetic Nomenclature (ISCN) ${ }^{10}$ and classified using the refined UK Medical Research Council (MRC) classification. ${ }^{6} \mathrm{MK}$ was defined as the presence of two or more autosomal monosomies or a single monosomy in the presence of structural abnormalities, as introduced by Breems et al. ${ }^{8}$

\section{Statistical analyses}

The duration of overall survival (OS) was calculated from the date of randomization until death. The Kaplan-Meier method was used to estimate the OS rates. ${ }^{11}$ Confidence intervals for the 5 -year OS rates were obtained using the normal approximation of the distribution of $\log [-\log ($ survival) $]$ and the Greenwood variance formula. ${ }^{12}$ The confidence interval of the median OS from CR/CRi was estimated based on the Brookmeyer and Crowley method. ${ }^{13}$ Log-rank tests and Cox models were used to compare OS between groups. ${ }^{14}$ Logistic regression was used to assess associations with CR/CRi achievement after induction. The analysis was stratified (in the case of survival analysis) or adjusted (in the case of logistic regression) by protocol when it included data from two trials. Multivariate Cox and logistic regression models were performed to assess the associations of MK and adverse MRC risk group with outcomes, adjusting for known prognostic factors. A Fisher exact test was used to investigate the association between two categorical variables. All reported $P$-values are two-sided. SAS 9.4 software (SAS Institute Inc. Cary, NC, USA) was used for the statistical analyses.

\section{Results}

\section{Patients}

In the AML-10 trial, 2,157 patients were randomized to receive daunorubicin, mitoxantrone or idarubicin. The current analyses were performed in a subgroup of 911 patients for whom cytogenetic data were available and who did not have $\mathrm{t}(8 ; 21)$, inv(16) or $\mathrm{t}(15 ; 17) ; 696$ of them were classified in the MRC intermediate cytogenetic risk group and 215 in the adverse group. Out of the 911 patients, 93 had a MK (5 with intermediate-risk cytogenetics and 88 with adverse risk) (Table 1, Figure 1). In the AML-12 trial, 1,942 patients were randomized between high-dose cytarabine or standard-dose cytarabine. The current analyses were performed in a subgroup of 1,079 patients for whom cytogenetic data were available and who did not have $\mathrm{t}(8 ; 21)$, inv(16) or $\mathrm{t}(15 ; 17) ; 896$ of them were classified in the MRC intermediate cytogenetic risk group and 182 in the adverse-risk group (information was missing for 1 patient). Out of these 1,079 patients, 95 had a MK (4 with intermediate-risk cytogenetics and 91 with adverse-risk) (Table 1, Figure 1). The patients' median follow up was 10.8 years in the whole population, 16.6 years among those in the AML-10 study and 9.9 years among AML-12 patients.

Monosomal karyotype is an independent poor prognostic factor in young acute myeloid leukemia patients

The impact of MK on AML outcomes was assessed by comparing outcomes of patients without $\mathrm{MK}$ and without 
adverse-risk cytogenetics (NotAdvMK-, $\mathrm{n}=1,584$ ), those without $\mathrm{MK}$ but with adverse-risk cytogenetics (AdvMK-, $\mathrm{n}=218)$ and those with a $\mathrm{MK}\left(\mathrm{MK}^{+}, \mathrm{n}=188\right)$.

\section{Achievement of complete response with or without blood count recovery}

Patients with AdvMK- [odds ratio (OR) $=1.80,95 \%$ confidence interval $(95 \% \mathrm{CI}): 1.33-2.42]$ or $\mathrm{MK}^{+}(\mathrm{OR}=3.09$, 95\% CI: 2.26-4.22) had a higher probability of not reaching a CR/CRi after induction compared to NotAdvMK patients (Table 2). CR/CRi was achieved in $76 \%, 63 \%$ and $50 \%$ of NotAdvMK-, AdvMK ${ }^{-}$and $\mathrm{MK}^{+}$patients, respectively. Comparing $\mathrm{MK}^{+}$to $\mathrm{MK}^{-}$patients (NotAdvMK- or AdvMK-), the odds of not achieving a CR/CRi were almost three times higher ( $\mathrm{OR}=2.85,95 \%$ CI: 2.10-3.88) for $\mathrm{MK}^{+}$patients. The probability of not achieving a $\mathrm{CR} / \mathrm{CRi}$ was also significantly higher in $\mathrm{MK}^{+}$than in AdvMK- patients (OR=1.72, 95\% CI: 1.15-2.57).

In a multivariate logistic regression model including age, WHO Performance Status, and white blood cell count, AdvMK- (OR 1.91, 95\% CI: 1.41-2.59) and $\mathrm{MK}^{+}$(OR 3.34, 95\% CI: 2.42-4.59) were associated with higher probabilities of not achieving a CR/CRi compared to NotAdvMK(Table 2).

\section{Overall survival}

The 5 -year OS rates were $39.1 \%, 24.1 \%$ and $7.2 \%$ in the NotAdvMK-, AdvMK- and $\mathrm{MK}^{+}$patients, respectively (Figure 2A). The estimated hazard ratios comparing AdvMK- and $\mathrm{MK}^{+}$patients to NotAdvMK- patients were 1.48 and 2.58, respectively (Table 3). Comparing $\mathrm{MK}^{+}$to $\mathrm{MK}^{-}$(NotAdvMK- or AdvMK') and AdvMK $\mathrm{K}^{-}$patients, the estimates of the unadjusted hazard ratio were 2.44 (95\% CI: 2.08-2.88) and 1.74 (95\% CI: 1.41-2.15), respectively.

In a multivariate Cox model, in comparison to NotAdvMK- patients, those with AdvMK- (HR 1.51, 95\% CI: $1.28-1.77$ ) or $\mathrm{MK}^{+}$(HR 2.71, 95\% CI: 2.29-3.20) had a shorter OS (Table 3).

\section{Overall survival from complete remission with or without hematologic recovery}

The 5-year OS rates from CR/CRi were 48.5\%, 35.5\% and $11.4 \%$ in NotAdvMK-, AdvMK- and $\mathrm{MK}^{+}$patients, respectively (Figure 2B). The estimated hazard ratios comparing AdvMK- and $\mathrm{MK}^{+}$patients to NotAdvMK- patients were 1.50 and 2.87, respectively (Table 4). Comparing $\mathrm{MK}^{+}$to $\mathrm{MK}^{-}$(NotAdvMK- or $\mathrm{AdvMK}^{-}$) and $\mathrm{AdvMK}^{-}$ patients, the estimates of the hazard ratio were $2.73(95 \%$ CI: $2.17-3.45)$ and 1.91 (95\% CI: 1.42-2.57), respectively.

In a multivariate Cox model, in comparison to NotAdvMK-, AdvMK- (HR 1.52, 95\% CI: 1.23-1.88) and $\mathrm{MK}^{+}$(HR 2.95, 95\% CI: 2.32-3.74) were associated with shorter OS from CR/CRi (Table 4). In a sensitivity analysis, we modified the multivariate model by additionally stratifying it by donor availability. The results of this analysis were similar to those of the main analysis ( $\mathrm{HR}=1.59$, 95\% CI: $1.28-1.98$ for $\mathrm{AdvMK}^{-}$versus NotAdvMK- and HR=3.00, 95\% CI: $2.35-3.82$, for $\mathrm{MK}^{+}$ versus NotAdvMK-).

\section{No impact of the type of anthracycline on outcomes in patients with a monosomal karyotype}

Response data were available for 91 out of $93 \mathrm{MK}^{+}$ patients from the AML-10 trial. CR/CRi was reached after induction by 18 out of 32 patients in the daunorubicin arm
(56\%), 14 out of 28 patients $(50 \%)$ in the mitoxantrone arm and 13 out of $31(42 \%)$ patients in the idarubicin arm $(P=0.54)$. The 5 -year OS rates were $13.0 \%$ (95\% CI: 4.1 $27.1 \%)$ in daunorubicin patients, $6.7 \%(95 \%$ CI: $1.2-$ $19.2 \%$ ) in mitoxantrone patients, and $11.7 \%$ (95\% CI: 3.1 $26.6 \%$ ) in idarubicin patients (Figure 2C). The 5-year OS rates from CR/CRi were $17.6 \%$ (95\% CI: $4.3-38.3 \%$ ) in daunorubicin patients, $11.5 \%$ (95\% CI: $0.9-37.5 \%)$ in idarubicin patients and $14.3 \%$ (95\% CI: $2.3-36.6 \%$ ) in mitoxantrone patients (logrank $P=0.53$ ).

\section{No benefit of high-dose cytarabine in patients with monosomal karyotype}

Response data after induction were available for 93 out of $95 \mathrm{MK}^{+}$and 978 out of $984 \mathrm{MK}^{-}$patients from the AML12 trial. MK (present vs. absent) was of predictive importance for the effect of high-dose cytarabine on the probability of reaching CR/CRi after induction (interaction test $P$-value: 0.01$). \mathrm{MK}^{-}$patients randomized to the high-dose cytarabine arm were more likely to reach CR/CRi com-

Table 1. Patients' characteristics.

\begin{tabular}{|c|c|c|c|}
\hline Study & NotAdvMK- & AdvMK- & MK+ \\
\hline N. of patients & 1,584 & 218 & 188 \\
\hline $\begin{array}{l}\text { MRC cytogenetic risk group, } \\
\text { N. of patients (\%) } \\
\text { Adverse } \\
\text { Intermediate } \\
\text { Missing }\end{array}$ & $\begin{array}{c}0 \\
1583(100) \\
1\end{array}$ & $\begin{array}{c}218(100) \\
0 \\
0\end{array}$ & $\begin{array}{c}179(5) \\
9(95) \\
0\end{array}$ \\
\hline $\begin{array}{l}\text { Trial } \\
\text { AML-10 } \\
\text { Daunorubicin, n. of patients (\%) } \\
\text { Idarubicin, n. of patients (\%) } \\
\text { Mitoxantrone, n. of patients (\%) } \\
\text { AML-12 } \\
\text { SDAC, n. of patients (\%) } \\
\text { HiDAC, n. of patients (\%) }\end{array}$ & $\begin{array}{c}691 \\
221(32.0) \\
226(32.7) \\
244(35.3) \\
893 \\
466(52.2) \\
427(47.8)\end{array}$ & $\begin{array}{c}127 \\
29(22.8) \\
56(44.1) \\
42(33.1) \\
91 \\
42(46.2) \\
49(53.8)\end{array}$ & $\begin{array}{c}93 \\
32(34.4) \\
31(33.3) \\
30(32.3) \\
\quad 95 \\
47(49.5) \\
48(50.5)\end{array}$ \\
\hline Male / Female, n. & $792 / 790$ & $107 / 111$ & $109 / 79$ \\
\hline $\begin{array}{l}\text { Age (years), n. of patients (\%) } \\
15-25 \\
26-45 \\
46-60\end{array}$ & $\begin{array}{l}155(10) \\
663(42) \\
766(48)\end{array}$ & $\begin{array}{c}28(13) \\
90(41) \\
100(46)\end{array}$ & $\begin{array}{l}15(8) \\
71(38) \\
102(54)\end{array}$ \\
\hline $\begin{array}{l}\text { WHO performance status, n. (\%) } \\
0 \\
1 \\
2-4 \\
\text { Missing }\end{array}$ & $\begin{array}{c}679(43) \\
672(42) \\
226(14) \\
7(0)\end{array}$ & $\begin{array}{l}84(39) \\
104(48) \\
30(14)\end{array}$ & $\begin{array}{l}62(33) \\
100(53) \\
26(14)\end{array}$ \\
\hline $\begin{array}{l}\text { WBC } \times 10^{9} / \mathrm{L} \text { at diagnosis, } n \text {. of pati } \\
<25 \\
25-99.9 \\
\geq 100 \\
\text { Missing }\end{array}$ & $\begin{array}{l}\text { ts }(\%) \\
892(56) \\
496(31) \\
195(12) \\
1(0)\end{array}$ & $\begin{array}{l}147(67) \\
51(23) \\
20(9) \\
0(0)\end{array}$ & $\begin{array}{l}140(75) \\
38(20) \\
10(5) \\
0(0)\end{array}$ \\
\hline $\begin{array}{l}\text { N. of patients with CR/CRi } \\
\text { after induction }\end{array}$ & 1194 & 137 & 92 \\
\hline $\begin{array}{l}\text { Donor, n. among pts with CR/CRi ( } \\
\text { No } \\
\text { Yes } \\
\text { Missing }\end{array}$ & $\begin{array}{c}710(59.5) \\
412(34.5) \\
72(6.0) \\
\end{array}$ & $\begin{array}{c}5(54.7) \\
58(42.3) \\
4(2.9) \\
\end{array}$ & $\begin{array}{r}55(59.8 \\
35(38.0 \\
2(2.2) \\
\end{array}$ \\
\hline
\end{tabular}

NotAdvMK: not adverse cytogenetic excluding a monosomal karyotype; AdvMKadverse cytogenetic excluding a monosomal karyotype; $\mathrm{MK}^{+}$: monosomal karyotype; SDAC, standard-dose cytarabine; HiDAC: high-dose cytarabine; WHO: World Health Organization; WBC: white blood cell count; CR: complete remission; CRi, complete remission with incomplete hematologic recovery. 
pared to those randomized to the standard-dose cytarabine arm $(\mathrm{OR}=1.51,95 \% \mathrm{CI}: 1.12-2.04$; CR/CRi rate $80 \%$ vs. $73 \%$, respectively). Among $\mathrm{MK}^{+}$patients, 19 (1 without hematologic recovery and 2 with missing hematologic recovery data) out of 46 patients from the high-dose cytarabine arm and 28 (5 without hematologic recovery and 1 with missing hematologic recovery data) out of 47 patients in the standard-dose cytarabine arm reached CR/CRi. In other words, in $\mathrm{MK}^{+}$patients the trend was even in a different direction $(\mathrm{OR}=0.48,95 \% \mathrm{CI}: 0.21-1.09$; CR/CRi rate $41 \%$ in the high-dose cytarabine arm vs. $60 \%$ in the standard-dose cytarabine arm, $P=0.080)$. Excluding six patients without hematologic recovery, the CR rate was $39 \%$ in the high-dose cytarabine arm versus $49 \%$ in the standard-dose cytarabine arm. Furthermore, among 38 patients with reported CR with a full hematologic recovery after induction, six (including 2 high-dose cytarabine and 4 standard-dose cytarabine patients) were reported not to have had a full hematologic recovery after consolidation.

Interestingly, no benefit of high-dose cytarabine on $O S$ was observed in the subgroup of $\mathrm{MK}^{+}$patients $(\mathrm{HR}=1.03$, 95\% CI: 0.68-1.57; $P=0.88$ ) (Figure 2D). The estimate of the hazard ratio for $\mathrm{OS}$ after CR/CRi among $\mathrm{MK}^{+}$patients was 0.82 (95\% CI: 0.44-1.53; $P=0.53)$.
AML-10
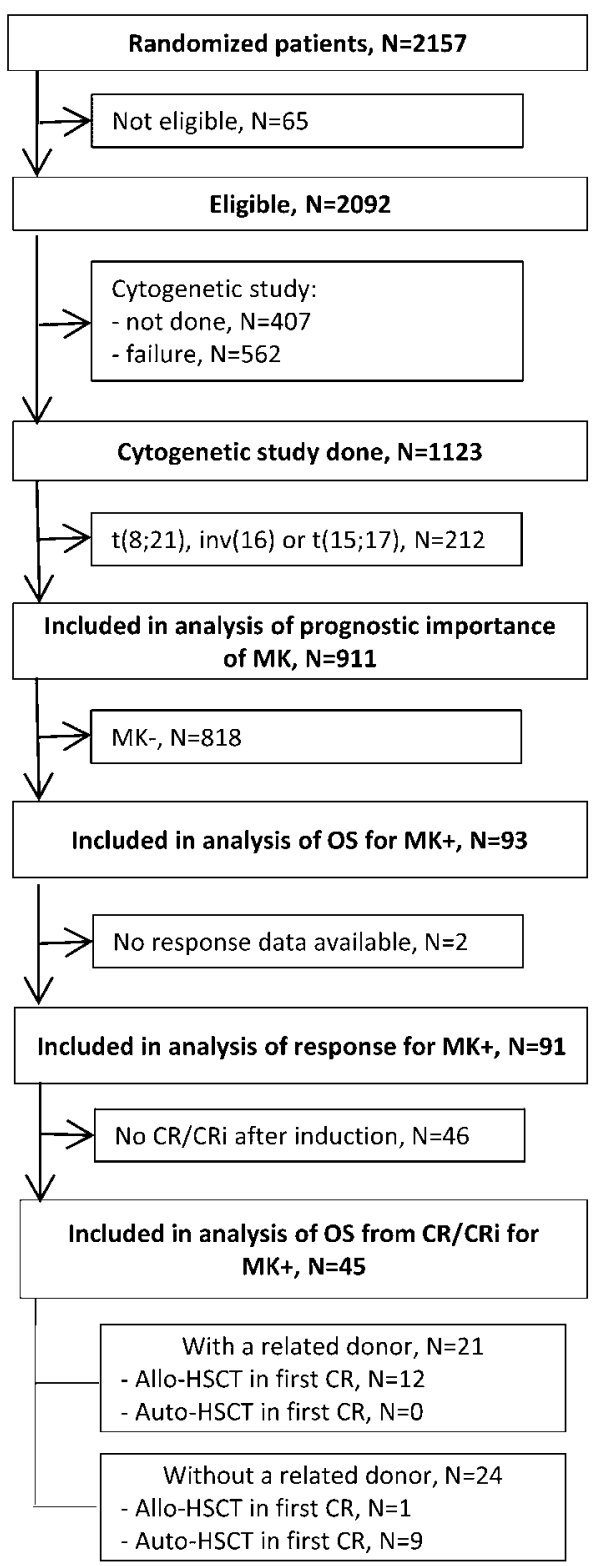

AML-12
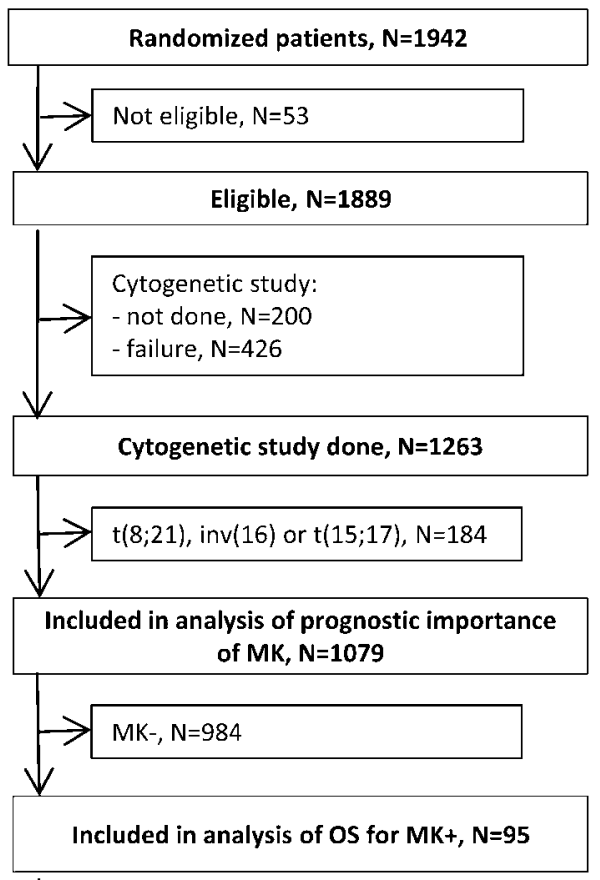

No response data available, $\mathrm{N}=2$<smiles>C1CC1</smiles>

Included in analysis of response for $\mathrm{MK}+, \mathrm{N}=93$

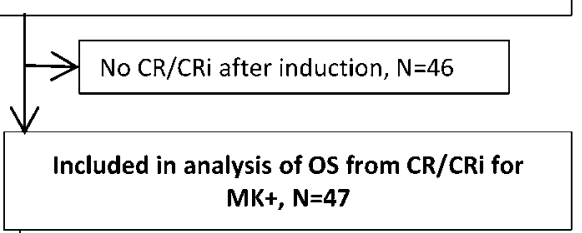

With a related donor, $\mathrm{N}=14$ - Allo-HSCT in first $\mathrm{CR}, \mathrm{N}=8$

Auto-HSCT in first $\mathrm{CR}, \mathrm{N}=1$

Without a related donor, $\mathrm{N}=31$ Allo-HSCT in first $\mathrm{CR}, \mathrm{N}=5$

- Auto-HSCT in first CR, N=13

With donor information missing, $\mathrm{N}=2$

Figure 1. Flow chart of the patients included in the current analyses. MK: monosomal karyotype (MK: without MK; MK': with MK); OS: overall survival; CR/Cri: complete remission/complete remission with incomplete blood count recovery; Allo-HSCT: allogeneic hematopoietic stem cell transplantation; Auto-HSCT: autologous hematopoietic stem cell transplantation. 
Having an HLA-identical related donor improved overall survival from complete remission in patients with a monosomal karyotype

A total of $92 \mathrm{MK}^{+}$patients achieved a CR/CRi on study. Among them, 35 patients had and 55 did not have an HLA-identical related donor (information was missing for 2 patients). Among those with an HLA related donor, 25 patients $(71 \%)$ received an allogeneic HSCT, including 20 $(57 \%)$ who were transplanted in first CR/CRi (Figure 1). In addition, 23 patients (including 22 without an HLAmatched related donor) underwent autologous HSCT in first CR/CRi.. The median time between CR/CRi and HSCT in first CR/CRi was 91 days for patients who received an autologous transplant (range, 42-167), 76 days for patients who received an allogeneic HSCT (range, 46171), 91 days for patients without a donor (range: 42.0 171.0) and 71 days for patients with a donor (range, 46.0 148.0). OS from CR/CRi was longer in patients with a donor than in those without, such that the 5 -year OS rates following CR/CRi were $24.1 \%$ (95\% CI: 11.4-39.3\%) and $3.8 \%$ (95\% CI: $0.7-11.5 \%)$, respectively ( $\mathrm{HR}=0.59,95 \%$ CI: 0.37-0.95) (Figure 3A).

Given the clinical importance of the observations described just above, we performed several sensitivity analyses to assess the impact of allogeneic HSCT on outcomes among $\mathrm{MK}^{+}$patients. The exclusion of patients with a CRi had little impact on the estimated hazard ratio ( $\mathrm{HR}=0.60,95 \% \mathrm{CI}: 0.37-0.98)$. The advantage of having an HLA-identical related donor remained present $(\mathrm{HR}=0.61$, 95\% CI: 0.38-0.99) after adjusting for patient's age (< or $\geq$ 45 years old).

Furthermore, the size of the estimated treatment effect confirmed the benefit of allogeneic HSCT when the posttransplant survival of patients who received an allogeneic graft was compared to that of patients who received an autologous graft ( $\mathrm{HR}=0.54,95 \%$ CI: 0.26-1.12) (Figure 3B). Second, in a Cox model including allogeneic HSCT (modeled as a time-varying covariate) and age, and stratified by protocol, allogeneic HSCT was associated with a longer survival from CR/CRi (HR=0.61, 95\% CI: 0.36-1.02).

\section{Discussion}

As demonstrated in this study, several other studies have established that the presence of a MK is associated with a particularly poor outcome in younger AML patients. , 15-17 $^{17}$ However, the number of studies regarding the best remission-induction regimens for $\mathrm{MK}^{+} \mathrm{AML}$ patients as well as the impact of allogeneic HSCT on the outcomes of such patients have been the focus of only a few studies. ${ }^{16,18}$ In order to investigate these important issues, we used the data concerning the $\mathrm{MK}^{+}$patients included in the EORTC/GIMEMA AML-10 and AML-12 phase III multicenter trials. Several observations were made.

First, our study confirms the poor prognosis associated with $\mathrm{MK}^{+}$in younger AML patients. Specifically, $\mathrm{MK}^{+}$was associated with a lower probability of achieving a CR/CRi, a shorter OS and a shorter OS from CR/CRi, whether taking other prognostic factors into account or not. This is in concordance with prior observations that the CR/CRi rate for adult $\mathrm{MK}^{+}$patients ranged from 14$43 \%$ and the OS rate from $9-18 \%{ }^{8,15-17}$

In addition, we investigated whether the type of anthra- cycline given during remission induction affected the outcomes of $\mathrm{MK}^{+}$patients among patients included in the AML-10 trial. Unfortunately, none of the assessed anthracyclines was associated with better outcomes among $\mathrm{MK}^{+}$ patients. These findings are consistent with the results of prior phase III trials showing that increasing the dose of daunorubicin was beneficial mainly in patients with favorable or intermediate-risk cytogenetics. ${ }^{1920}$

In the first analysis of the AML-12 trial, we observed that induction with high-dose cytarabine increased the proportion of patients achieving a CR/CRi and prolonged OS in patients younger than 46 years of age. ${ }^{1}$ This benefit was also observed in patients with adverse cytogenetic abnormalities and/or FLT3-internal tandem duplication (ITD) mutations, as well as in those with secondary AML. Here we found no evidence of a benefit of high-dose cytarabine in the subgroup of patients with a MK. There was even a suggestion of a lower incidence of CR/CRi in $\mathrm{MK}^{+}$patients randomized to the high-dose cytarabine arm. This finding could be explained by previous studies showing that up to $80 \%$ of $\mathrm{MK}^{+}$patients have a mutation in the TP53 gene ${ }^{21}$ Patients with TP53 mutation in their tumor cells are resistant to high-dose cytarabine, as has been demonstrated in patients with mantle cell lymphoma. ${ }^{22}$ Unfortunately, the TP53 gene mutation was not evaluated in the present study.

Finally, we investigated whether patients with a donor had an OS benefit in comparison to those without a donor. As previously demonstrated by us and by other groups of investigators, among MK- AML patients with intermediate or unfavorable karyotype, the presence of a

Table 2. Association between monosomal karyotype and Medical Research Council adverse-risk group and achievement of complete remission with or without hematologic recovery after induction.

\begin{tabular}{|c|c|c|c|}
\hline Covariate & OR of no CR/CRi & $95 \%$ CI & $P$ value \\
\hline \multicolumn{4}{|c|}{ Unadjusted analysis $^{\wedge}$} \\
\hline $\begin{array}{l}\text { Cytogenetic group } \\
\text { NotAdvMK } \\
\text { AdvMK } \\
\mathrm{MK}^{+}\end{array}$ & $\begin{array}{c}1 \\
1.80 \\
3.09\end{array}$ & $\begin{array}{l}1.33-2.42 \\
2.26-4.22\end{array}$ & $<0.001$ \\
\hline \multicolumn{4}{|c|}{ Multivariate analysis* } \\
\hline $\begin{array}{l}\text { Cytogenetic group } \\
\text { NotAdvMK } \\
\text { AdvMK } \\
\mathrm{MK}^{+}\end{array}$ & $\begin{array}{c}1 \\
1.91 \\
3.34\end{array}$ & $\begin{array}{l}1.41-2.59 \\
2.42-4.59\end{array}$ & $<0.001$ \\
\hline $\begin{array}{l}\text { Age (years) } \\
15-25 \\
26-45 \\
46-60\end{array}$ & $\begin{array}{c}1 \\
1.35 \\
1.61\end{array}$ & $\begin{array}{l}0.92-1.99 \\
1.10-2.36\end{array}$ & 0.028 \\
\hline $\begin{array}{l}\text { WHO Performance } \\
0 \\
1 \\
2-4\end{array}$ & $\begin{array}{r}\text { us at baseline } \\
1 \\
1.07 \\
1.83\end{array}$ & $\begin{array}{l}0.85-1.34 \\
1.35-2.48\end{array}$ & $<0.001$ \\
\hline $\begin{array}{l}\text { WBC at diagnosis } \\
<25 \\
\geq 25 \text { and }<100 \\
\geq 100\end{array}$ & $\begin{array}{c}1 \\
1.21 \\
1.79\end{array}$ & $\begin{array}{l}0.96-1.52 \\
1.31-2.46\end{array}$ & 0.001 \\
\hline
\end{tabular}

OR: odds ratio; $95 \%$ Cl: 95\% confidence interval; NotAdvMK-: not adverse cytogenetic excluding a monosomal karyotype; AdvMK-: adverse cytogenetic excluding a monosomal karyotype; MK': monosomal karyotype;WHO:World Health Organization;WBC: white blood cell count. 'Obtained with a logistic regression model including protocol (AML-10 vs. AML-12) and cytogenetic group. *Obtained with a logistic regression model including protocol and all covariates presented in the Table. 
donor for an allogeneic HSCT prolonged OS., ${ }^{7,23}$ Interestingly, we made similar findings in patients with $\mathrm{MK}^{+}$, suggesting a positive impact of allogeneic HSCT in this population of patients with a generally poor outcome. To our knowledge, our study is the first to investigate the impact of allogeneic HSCT in $\mathrm{MK}^{+}$patients using a donor versus no donor comparison (which is considered as the gold-standard technique to address this question when donor availability is prospectively collected). These results were further confirmed in a Cox model handling allogeneic HSCT as a time-varying covariate and by comparing patients who received an allogeneic transplant from an HLA-matched related donor with those who received an autologous transplant. The three approaches, which are based on different assumptions, consistently indicated a positive impact of allogeneic HSCT. Among 20 patients who underwent allogeneic HSCT from an HLA-matched related donor, two were still alive and in follow-up 10 years after reaching $C R$, indicating a curative potential of the treatment. Importantly, our results are concordant with prior results from Kayser et al., who observed that allo- geneic HSCT prolonged OS in younger (18-60 years of age) $\mathrm{MK}^{+} \mathrm{AML}$ patients (using Mantel-Byar analysis) ${ }^{13}$, as well as with a recent publication by Cornelissen et al., who demonstrated better leukemia-free survival in younger $\mathrm{MK}^{+}$AML patients offered allogeneic HSCT (using a timedependent Cox analysis). ${ }^{18}$ This is also concordant with previous studies showing that, although the prognosis of $\mathrm{MK}^{+}$patients remains worse than that of $\mathrm{MK}^{-}$patients after transplantation, they have a 3-4 year OS probability ranging from $25 \%$ to $34 \%{ }^{24,25}$ Interestingly, a study by the Acute Leukemia Working Party of the European Society for Blood and Marrow Transplantation observed relatively comparable relapse incidence ( $\mathrm{HR}=1.3$, 95\% CI: 0.7-2.6) and $\mathrm{OS}(\mathrm{HR}=0.9,95 \% \mathrm{CI}: 0.5-1.6)$ in $\mathrm{MK}^{+}$patients given grafts after reduced-intensity or myeloablative conditioning. ${ }^{25}$ This suggests that cure of $\mathrm{MK}^{+}$patients after allogeneic HSCT might depend on immune-mediated graft-versustumor effects rather than on the intensity of the conditioning regimen. ${ }^{26}$

In summary, this retrospective analysis of two large prospective phase III trials confirmed the poor outcome of
A
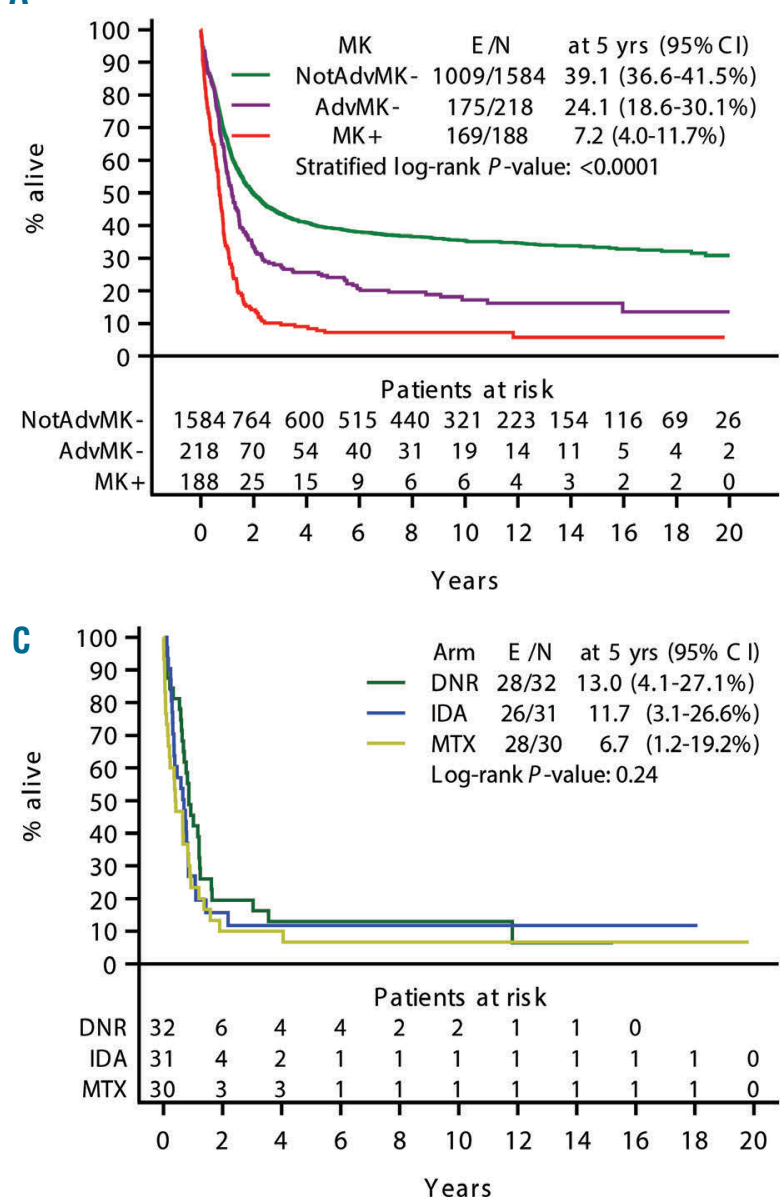

B

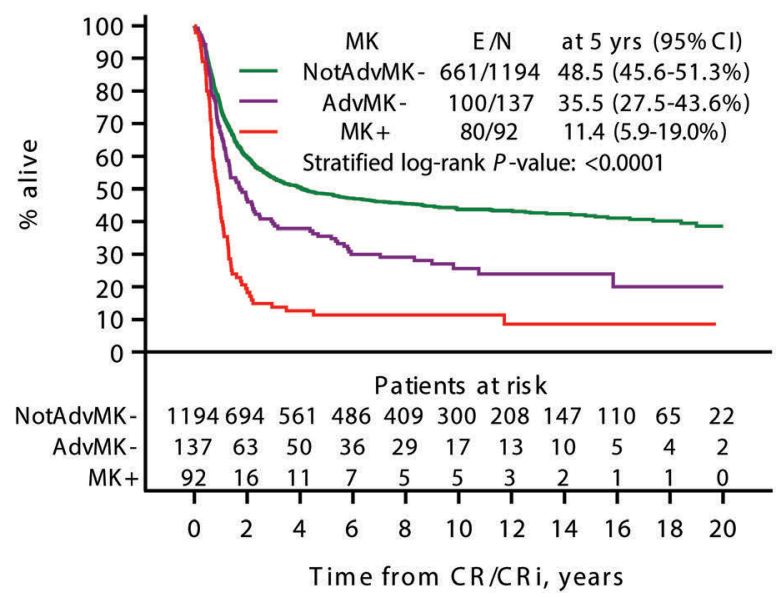

D

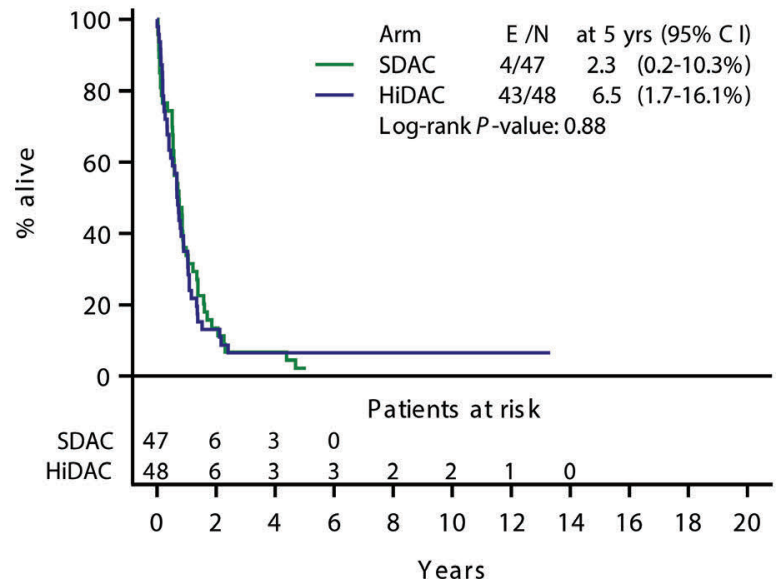

Figure 2. Impact of cytogenetic risk on overall survival and impact of randomization in patients with a monosomal karyotype. (A) Overall survival (OS) according to cytogenetic risk group. (B) OS from complete remission/complete remission with incomplete blood count recovery according to cytogenetic risk group. (C) OS according to randomized induction therapy in the AML-10 trial among patients with a monosomal karyotype. (D) OS according to randomized induction therapy in the AML-12 trial among patients with a monosomal karyotype. MK: monosomal karyotype; 95\% Cl: 95\% confidence interval; NotAdvMK: not adverse cytogenetics excluding a monosomal karyotype; AdvMK־: adverse cytogenetics excluding a monosomal karyotype; MK': monosomal karyotype.; DNR: daunorubicin; IDA: idarubicin; MTX: Methotrexate. 
Table 3. Association between monosomal karyotype and Medical Research Council adverse-risk group and overall survival.

\begin{tabular}{|c|c|c|c|}
\hline Covariate & HR & $95 \%$ CI & $P$ value \\
\hline \multicolumn{4}{|c|}{ 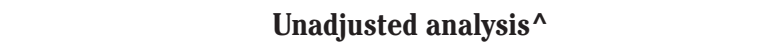 } \\
\hline $\begin{array}{l}\text { Cytogenetic group } \\
\text { NotAdvMK } \\
\text { AdvMK } \\
\mathrm{MK}^{+}\end{array}$ & $\begin{array}{c}1 \\
1.48 \\
2.58\end{array}$ & $\begin{array}{l}1.26-1.74 \\
2.19-3.04\end{array}$ & $<0.001$ \\
\hline \multicolumn{4}{|c|}{ Multivariate analysis* } \\
\hline $\begin{array}{l}\text { Cytogenetic group } \\
\text { NotAdvMK- } \\
\text { AdvMK } \\
\mathrm{MK}^{+}\end{array}$ & $\begin{array}{c}1 \\
1.51 \\
2.71\end{array}$ & $\begin{array}{l}1.28-1.77 \\
2.29-3.20\end{array}$ & $<0.001$ \\
\hline $\begin{array}{l}\text { Age (years) } \\
15-25 \\
26-45 \\
46-60\end{array}$ & $\begin{array}{c}1 \\
1.05 \\
1.44\end{array}$ & $\begin{array}{l}0.86-1.29 \\
1.18-1.75\end{array}$ & $<0.001$ \\
\hline $\begin{array}{l}\text { WHO Performance Status a } \\
0 \\
1 \\
2-4\end{array}$ & $\begin{array}{c}\text { seline } \\
1 \\
1.09 \\
1.76\end{array}$ & $\begin{array}{l}0.97-1.23 \\
1.50-2.07\end{array}$ & $<0.001$ \\
\hline $\begin{array}{l}\text { WBC at diagnosis }\left(\times 10^{9} / \mathrm{L}\right) \\
<25 \\
\geq 25 \text { and }<100 \\
\geq 100\end{array}$ & $\begin{array}{c}1 \\
1.08 \\
1.42\end{array}$ & $\begin{array}{l}0.95-1.22 \\
1.20-1.68\end{array}$ & $<0.001$ \\
\hline
\end{tabular}

HR: hazard ratio; $95 \%$ CI: $95 \%$ confidence interval; NotAdvMK: not adverse cytogenetic excluding a monosomal karyotype; AdvMK:- adverse cytogenetic excluding a monosomal karyotype; $\mathrm{MK}^{\star}$ : monosomal karyotype; WHO:World Health Organization WBC: white blood cell count. 'Obtained with a logistic regression model including protocol (AML-10 vs.AML-12) and cytogenetic group. * ${ }^{*}$ Obtained with a logistic regression model including protocol and all covariates presented in the Table.
Table 4. Association between monosomal karyotype and Medical Research Council adverse-risk group and overall survival from complete remission with or without hematologic recovery.

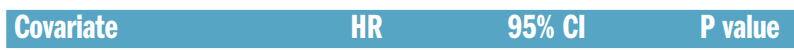

$\begin{array}{lcr}\text { Cytogenetic group } & & \\ \text { NotAdvMK } & 1 & \\ \text { AdvMK } & 1.50 & 1.22-1.85 \\ \text { MK }^{+} & 2.87 & 2.27-3.63\end{array}$

Multivariate analysis*

$\begin{array}{lcc}\text { Cytogenetic group } & & \\ \text { NotAdvMK } & 1 & - \\ \text { AdvMK } & 1.52 & 1.23-1.88 \\ \text { MK }^{+} & 2.95 & 2.32-3.74\end{array}$

\begin{tabular}{lccc} 
Age (years) & & & $<0.001$ \\
$15-25$ & 1 & & \\
$26-45$ & 1.05 & $0.82-1.35$ & \\
$46-60$ & 1.46 & $1.15-1.86$ & \\
\hline WHO Performance Status at baseline & & 0.004
\end{tabular}

$\begin{array}{lcc}0 & 1 & \\ 1 & 1.06 & 0.92-1.23 \\ 2-4 & 1.43 & 1.16-1.78\end{array}$

\begin{tabular}{lcc}
$\mathrm{WBC}$ at diagnosis $\left(\mathrm{x} 10^{9} / \mathrm{L}\right)$ & & \\
$<25$ & 1 & \\
$\geq 25$ and $<100$ & 1.00 & 0.069 \\
$\geq 100$ & 1.30 & $1.03-1.17$ \\
\hline
\end{tabular}

HR: hazard ratio; $95 \%$ CI: $95 \%$ confidence interval; NotAdvMK $:$ not adverse cytogenetic excluding a monosomal karyotype; AdvMK: adverse cytogenetic excluding a monosomal karyotype; $\mathrm{MK}^{+}$: monosomal karyotype;WHO:World Health Organization WBC: white blood cell count. ^ Obtained with a logistic regression model including protocol (AML-10 vs.AML-12) and cytogenetic group. * Obtained with a logistic regression model including protocol and all covariates presented in the Table.

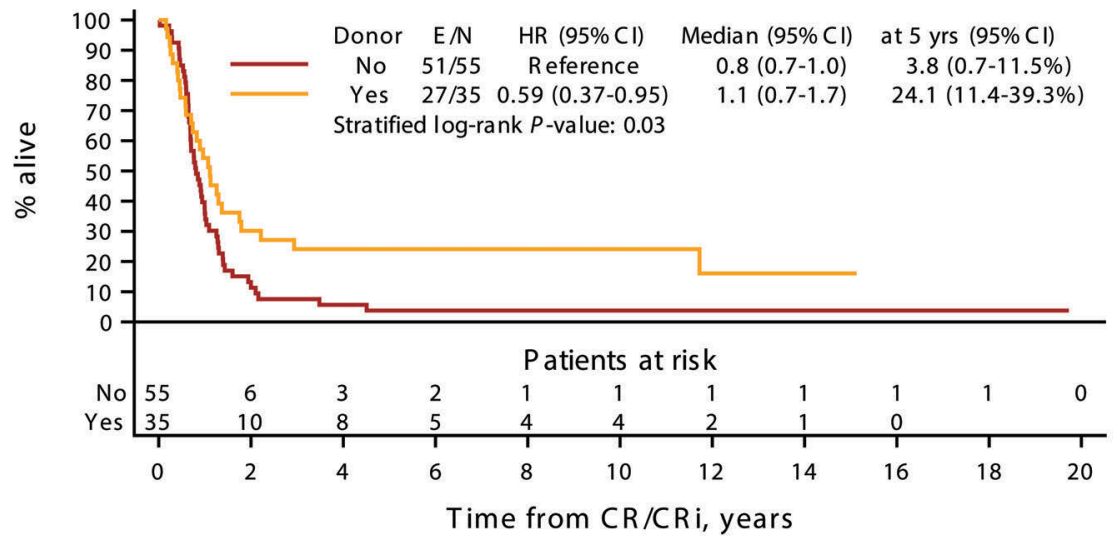

B

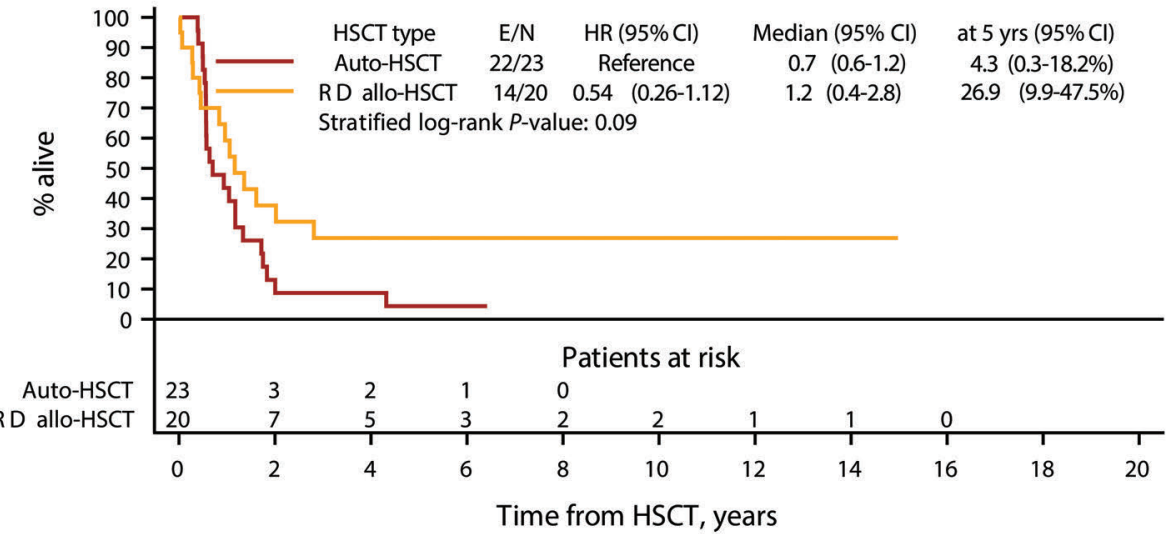

Figure 3. Impact of allogeneic hematopoietic stem cell transplantation in patients with a monosomal karyotype. (A) Overall survival from complete remission/complete remission with incomplete blood count recovery according to donor availability among patients with a monosomal karyotype. (B) Overall survival from hematopoietic stem cell transplantation by type of transplant among patients with monosomal karyotype. HR: hazard ratio; 95\% Cl: 95\% confidence interval; CR/Cri: complete remission/complete remission with incomplete blood count recovery; HSCT: hematopoietic stem cell transplantation; Auto-; autologous: RD allo-: related donor allogeneic. 
$\mathrm{MK}^{+}$patients. We found no evidence that the outcome was affected by the type of remission-induction chemotherapy. Donor availability was associated with prolonged survival among patients who reached a CR/CRi, suggesting a positive effect of allogeneic HSCT in this population of patients. These findings highlight the need for further prospective studies assessing the best strategy to bring $\mathrm{MK}^{+}$patients to an allogeneic HSCT. In addition, efforts should be made to prevent post-transplant relapse and decrease transplant-related mortality in
$\mathrm{MK}^{+} \mathrm{AML}$ patients. One possible strategy to do this could be post-transplantation prophylactic administration of hypomethylating agents, given their anti-leukemic activity in $\mathrm{MK}^{+}$patients ${ }^{27}$ and their ability to prevent graft-versus-host disease..$^{28}$

\section{Funding}

This publication was supported by a donation from the "Fondation Contre le Cancer" from "Belgium" through the EORTC Cancer Research Fund.

\section{References}

1. Willemze R, Suciu S, Meloni G, et al. Highdose cytarabine in induction treatment improves the outcome of adult patients younger than age 46 years with acute myeloid leukemia: results of the EORTCGIMEMA AML-12 Trial. J Clin Oncol. 2014:32(3):219-228.

2. Mandelli F, Vignetti M, Suciu S, et al. Daunorubicin versus mitoxantrone versus idarubicin as induction and consolidation chemotherapy for adults with acute myeloid leukemia: the EORTC and GIMEMA groups study AML-10. J Clin Oncol. 2009;27(32):5397-5403.

3. Burnett AK, Russell NH, Hills RK, et al. Optimization of chemotherapy for younger patients with acute myeloid leukemia: results of the Medical Research Council AML15 trial. J Clin Oncol. 2013;31(27):33603368.

4. Walter RB, Othus M, Burnett AK, et al. Resistance prediction in AML: analysis of 4601 patients from MRC/NCRI, HOVON/SAKK, SWOG and MD Anderson Cancer Center. Leukemia. 2015;29(2):312320.

5. Dohner H, Estey EH, Amadori S, et al. Diagnosis and management of acute myeloid leukemia in adults: recommendations from an international expert panel, on behalf of the European LeukemiaNet. Blood. 2010;115(3):453-474.

6. Grimwade D, Hills RK, Moorman AV, et al. Refinement of cytogenetic classification in acute myeloid leukemia: determination of prognostic significance of rare recurring chromosomal abnormalities among 5876 younger adult patients treated in the United Kingdom Medical Research Council trials. Blood. 2010;116(3):354-365.

7. Suciu S, Mandelli F, de Witte $\mathrm{T}$, et al. Allogeneic compared with autologous stem cell transplantation in the treatment of patients younger than 46 years with acute myeloid leukemia (AML) in first complete remission (CR1): an intention-to-treat analysis of the EORTC/GIMEMAAML-10 trial. Blood. 2003;102(4):1232-1240.

8. Breems DA, van Putten WL, De Greef GE, et al. Monosomal karyotype in acute myeloid leukemia: a better indicator of poor progno- sis than a complex karyotype. J Clin Oncol. 2008;26(29):4791-4797.

9. Cornelissen JJ, Versluis J, Passweg JR, et al. Comparative therapeutic value of postremission approaches in patients with acute myeloid leukemia aged 40-60 years. Leukemia. 2014:29(5):1041-1050

10. Simons A, Shaffer LG, Hastings RJ Cytogenetic nomenclature: changes in the ISCN 2013 compared to the 2009 edition. Cytogenet Genome Res. 2013;141(1):1-6.

11. Kaplan EL, Meier P. Nonparametric estimation from incomplete observations. J Am Stat Assoc. 1958:53:457-481.

12. Greenwood M. The natural duration of cancer. Rep Public Health Med Subj 1926;33:126.

13. Kalbfleisch JD, Prentice RL. The statistical analysis of failure time data. John Wiley, 2002.

14. Cox DR. Regression models and life tables (with discussion). J R Stat Soc Series B. 1972;34(2):187-220

15. Medeiros BC, Othus M, Fang M, Roulston D, Appelbaum FR. Prognostic impact of monosomal karyotype in young adult and elderly acute myeloid leukemia: the Southwest Oncology Group (SWOG) experience. Blood. 2010;116(13):2224-2228.

16. Kayser S, Zucknick M, Dohner K, et al. Monosomal karyotype in adult acute myeloid leukemia: prognostic impact and outcome after different treatment strategies. Blood. 2012;119(2):551-558

17. Yanada M, Kurosawa S, Yamaguchi T, et al. Prognosis of acute myeloid leukemia harboring monosomal karyotype in patients treated with or without allogeneic hematopoietic cell transplantation after achieving complete remission. Haematologica. 2012;97(6):915918.

18. Cornelissen JJ, Breems D, van Putten WL, et al. Comparative analysis of the value of allogeneic hematopoietic stem-cell transplantation in acute myeloid leukemia with monosomal karyotype versus other cytogenetic risk categories. J Clin Oncol. 2012;30(17): 2140-2146.

19. Fernandez HF, Sun Z, Yao X, et al. Anthracycline dose intensification in acute myeloid leukemia. N Engl J Med. 2009;361(13):1249-1259.

20. Lowenberg B, Ossenkoppele GJ, van Putten W, et al. High-dose daunorubicin in older patients with acute myeloid leukemia. N
Engl J Med. 2009;361(13):1235-1248.

21. Rucker FG, Schlenk RF, Bullinger L, et al TP53 alterations in acute myeloid leukemia with complex karyotype correlate with specific copy number alterations, monosomal karyotype, and dismal outcome. Blood. 2012;119(9):2114-2121.

22. Delfau-Larue MH, Klapper W, Berger F, et al. High-dose cytarabine does not overcome the adverse prognostic value of CDKN2A and TP53 deletions in mantle cell lymphoma. Blood. 2015;126(5):604-611

23. Cornelissen JJ, van Putten WL, Verdonck LF et al. Results of a HOVON/SAKK donor versus no-donor analysis of myeloablative HLA-identical sibling stem cell transplantation in first remission acute myeloid leukemia in young and middle-aged adults: benefits for whom? Blood. 2007;109(9): 3658-3666.

24. Fang M, Storer B, Estey E, et al. Outcome of patients with acute myeloid leukemia with monosomal karyotype who undergo hematopoietic cell transplantation. Blood. 2011;118(6):1490-1494.

25. Poire X, Labopin M, Cornelissen JJ, et al Outcome of conditioning intensity in acute myeloid leukemia with monosomal karyotype in patients over 45 year-old: a study from the Acute Leukemia Working Party (ALWP) of the European Group of Blood and Marrow Transplantation (EBMT). Am J Hematol. 2015;90(8):719-724.

26. Baron $\mathrm{F}$, Labopin $M$, Niederwieser $D$, et al Impact of graft-versus-host disease after reduced-intensity conditioning allogeneic stem cell transplantation for acute myeloid leukemia: a report from the Acute Leukemia Working Party of the European group for blood and marrow transplantation. Leukemia. 2012;26(12):2462-2468

27. Lubbert M, Suciu S, Hagemeijer A, et al Decitabine improves progression-free survival in older high-risk MDS patients with multiple autosomal monosomies: results of a subgroup analysis of the randomized phase III study 06011 of the EORTC Leukemia Cooperative Group and German MDS Study Group. Ann Hematol. 2016;95(2):191-199.

28. Fransolet G, Ehx G, Somja J, et al. Azacytidine mitigates experimental sclerodermic chronic graft-versus-host disease. J Hematol Oncol. 2016;9(1):53. 